\title{
EARTH MATERIALS AT HIGH PRESSURE AND TEMPERATURE: RECENT CONTRIBUTIONS FROM IN SITU SYNCHROTRON X-RAY DIFFRACTION
}

\author{
S.A.T. REDFERN \\ Department of Earth Sciences, University of Cambridge \\ Downing Street, Cambridge, CB2 3EQ, United Kingdom
}

\begin{abstract}
The application of synchrotron X-ray powder diffraction to the study of minerals and understanding of the structure and dynamics of the Earth's deep interior is summarised. The salient features of the diamond anvil cell and large-volume multi-anvil cell high- $P / T$ apparatus as used at synchrotron radiation sources are described, and recent developments and applications of these techniques within high- $P / T$ mineralogy are reviewed.
\end{abstract}

PACS numbers: $07.35 .+k, 64.60 .-\mathrm{i}, 91.35 .-\mathrm{x}$

\section{Introduction}

An appreciation of mineral behaviour under the high pressure $(P)$ and temperature $(T)$ conditions of planetary interiors is central to understanding the structure and dynamics of the Earth's interior. The elastic properties of the postulated mineral assemblages of the Earth's mantle, for example, can be associated with the observed seismic data, thus forming a link between the seismologically-derived density-depth profile of our planet to the pressure-volume behaviour of the potential mantle minerals determined by in situ high- $P / T$ crystallography. Pressuretemperature phase diagrams can be constructed for the mineral assemblages of presumed relevance, using the equations of state of these phases. Hence, high- $P / T$ in situ X-ray diffraction can be used to test the stability limits of mineral phases which form part of particular high- $P / T$ assemblages. This can help identify the sources and sinks of geochemically-important elements within the planet's interior, as has recently been demonstrated from high- $P$ synchrotron powder diffraction of magnesite, $\mathrm{MgCO}_{3}$, which indicates that this phasc is the likely host for carbon throughout the Earth's mantle [1]. Much work of this type is currently focused on two areas of the Earth's interior, attempting to quantify the extent of incorporation of $\mathrm{H}_{2} \mathrm{O}$ and other volatile species in minerals which are subducted to relatively low depths as part of the oceanic crust at ocean margins [2,3], and attempting 
to elucidate the likely extent of incorporation of candidate light elements as alloys and Fe-rich compounds in the solid Fe-rich core $[4,5]$. Such studies have placed important constraints on the geochemical models of the Earth.

In addition to simply enabling the charting and calculation of phase diagrams, in situ diffraction studies have also led to an increased awareness of the importance of structural phase transitions in controlling planetary processes. Such transformations, occurring within minerals as a response to changing $P$ and $T$, can result in dramatic changes in physical properties (e.g. density, heat flow, viscosity, electrical conduction mechanisms, and geomagnetic properties). For example, the density changes associated with the transformations between $(\mathrm{Mg}, \mathrm{Fe})_{2} \mathrm{SiO}_{4}$ polymorphs from the olivine structure $\left(\alpha-(\mathrm{Mg}, \mathrm{Fe})_{2} \mathrm{SiO}_{4}\right)$ to wadsleyite $\left(\beta-(\mathrm{Mg}, \mathrm{Fe})_{2} \mathrm{SiO}_{4}\right.$, a defect spinel $)$ to ringwoodite $\left(\gamma-(\mathrm{Mg}, \mathrm{Fe})_{2} \mathrm{SiO}_{4}\right.$, a spinel phase) appear to account for much of the observed seismic discontinuity at $410 \mathrm{~km}$ depth (the top of the transition zone). At the base of the transition zone the reaction $\gamma-(\mathrm{Mg}, \mathrm{Fe})_{2} \mathrm{SiO}_{4 \text { (ringwoodite) }} \leftrightarrow \mathrm{MgSiO}_{3 \text { (silicate perovskite) }}+\mathrm{MgO}_{\text {(magnesiowüstite) }}$ appears to correlate (both in density change and the position of the $P / T$ phase boundary) with an observed discontinuity in seismic wave velocities at $670 \mathrm{~km}$ depth, the boundary between the transition zone and the lower mantle. Mineral physics has had a large input into our interpretation of the seismic properties of the upper mantle, transition zone, and lower mantle. High $P / T$ synchrotron in situ studies have played a leading role in this input. Now geophysicists have begun to consider not only the controls of phase transitions on seismic properties, but also their controls on heat flow from the core. Mineral phase transitions are found to have profound implications for the patterns of convection in the mantle, and it seems from recent numerical modelling that the transformations of phases in the $(\mathrm{Mg}, \mathrm{Fe})_{2} \mathrm{SiO}_{4}$ system determine the depth-dependence of the dominant mechanisms of mantle convection [6]. There are clearly important inter-relations between high- $P / T$ mineralogy, geophysics, and geochemistry. In forming unified compositional-thermal-seismological models of the Earth there is a pressing need for a reliable and accurate understanding of the properties of Earth materials with $P$ and $T$, and over the last two decades this need has been addressed principally by synchrotron X-ray diffraction studies using both large-volume (LV) apparatus and diamond anvil cell (DAC) techniques.

Here, we shall introduce the essential features of the main high- $P / T$ synchrotron techniques of relevance to the Earth Sciences, and discuss recent advances in their implementation as well as the potential limitations of their use. We will illustrate their application by briefly reviewing some recent studies conducted using them, and indicate possible directions for their future use.

\section{Diamond anvil cell studies}

Diamond anvil cells have been used extensively in the solution of Earth Science problems, by both single crystal and powder X-ray diffraction methods. Synchrotron radiation has been employed principally in powder diffraction studies using energy dispersive diffraction, taking advantage of the white radiation characteristics of the synchrotron source. In this geometry the diffracted rays are detected at a fixed $2 \theta$ from a powdered sample held in the DAC. Since there is no 
need for movement of any of the apparatus during the experiment the geometrical constraints on the design of the cell are not as great as they are for a single crystal diffraction experiment, and it is possible to attain very high pressures using lever-arm devices, compared with only moderate pressures (nevertheless now exceeding $15 \mathrm{GPa}$ or more) in single crystal experiments. A further drawback of the single crystal technique for very many Earth Science problems is, however, the unavailability of suitable single crystals for study, and the prevalence of high pressure ferroelastic twinning. Transformation twinning has been noted now in many Earth materials and when it occurs it renders single crystal techniques effectively useless for study of the high pressure phases.

Because of the relatively poor energy-resolution of energy-sensitive detectors and the fact that (usually) only part of the diffracted cone of intensity is detected, the precision and accuracy of energy-dispersive diffraction for the determination of high pressure cell parameters is, however, around an order of magnitude or more worse than the best and latest laboratory single crystal methods. An alternative is to use angle-dispersive methods. By selecting hard monochromatic X-rays it remains possible to collect diffraction patterns to reasonably small $d$-spacings within the restricted $2 \theta$ opening of the conventional cell design (which generally limits $2 \theta$ to around 40 or $45^{\circ}$ at best). Linear angle-dispersive detectors have been employed at NSLS by Jephcoat and co-workers in some very-high-pressure studies of Earth materials [7], but by using image plates the entire cones of the diffracted beams can be integrated and superior results obtained, so far at more moderate pressures [8]. Using image-plate geometries cell parameters can be obtained to within half an order of magnitude of the equivalent single-crystal experiment, and integration of the whole powder ring means that intensities can be obtained with some reliability, realistically allowing the possibility of whole-pattern structure refinement.

As well as the errors in cell parameters obtained from high-pressure experiments, in the calculation of equations of state further errors are introduced due to the uncertainties in pressure determination, whether they are obtained from the measurement of ruby fluorescence or from the compression of internal standards mixed with the sample. A recent and promising high-temperature lower-pressure. (up to $1 \mathrm{GPa}$ ) development of the DAC technique has been the use of the hydrothermal DAC, in which water is used as pressure-transmitting medium and a vapour bubble is incorporated within the sample chamber along with the sample. Knowing the liquid-vapour boundary in $P / T$ space, and observing the temperature of homogenisation of the vapour bubble within the DAC, it is possible to determine $P$ directly from $T$ as the DAC is taken along $P / T$ paths corresponding to isochores, using externally-heated DACs. The determination of pressure using this technique can be done with an order of magnitude or more better precision than ruby fluorescence or internal standard methods. In this way Bassett and co-workers have studied the (modestly) high- $P / T$ dehydration of swelling clays [9] and have also defined a $P / T$ standard using the anisotropic behaviour of the cell parameters of calcite [10]. In both cases they used energy-dispersive diffraction as the crystallographic probe. The $P / T$ limits of this technique are set in $T$-space by the burning of diamond, and in $P$-space by the compressional properties of $\mathrm{H}_{2} \mathrm{O}$. In effect, the technique amounts to a low- $P$ extension of high- $T$ methods. 
The processes of clay dehydration are important in low-temperature metamorphism in near-surface sedimentary environments of the Earth's crust. The other area of development in combined high $P$ and $T$ DAC techniques has been in the use of laser-heated DACs, corresponding to the opposite extreme of high- $P / T$ mineralogy from the hydrothermal DAC work. Here a Nd-YAG laser is focused onto the sample within the gasket and the temperatures and pressures of the experiment are generally not determined with any certainty. In addition, the $P / T$ gradients within the cells are generally poorly constrained, and these limitations mean that the data are rarely reliable enough to calculate thermodynamic properties with the sort of accuracy and consistency expected of lower- $P / T$ data sets. None the less, this does provide the only way of approaching the $P / T$ conditions of the core in situ, and has, for example, been employed recently in an investigation of Fe which suggested the possible existence within the Earth's core of a new phase of $\mathrm{Fe}$, a double-cell hcp polytype, which has not previously been considered in geophysical models of the core [11]. Such studies push the interpretation of high- $P / T$ energy-dispersive data to its limits. An example of the use of image plates in high- $P / T$ mineralogical DAC powder diffraction is afforded by a recent study of the high- $P$ low- $T$ metastable phases of $\mathrm{CaCO}_{3}$, conducted by the author and co-workers [12]. Calcium carbonate crystallises naturally as either rhombohedral calcite-I or as orthorhombic aragonite. In one of the earliest high-pressure mineralogical studies Bridgman [13] observed that calcite transforms
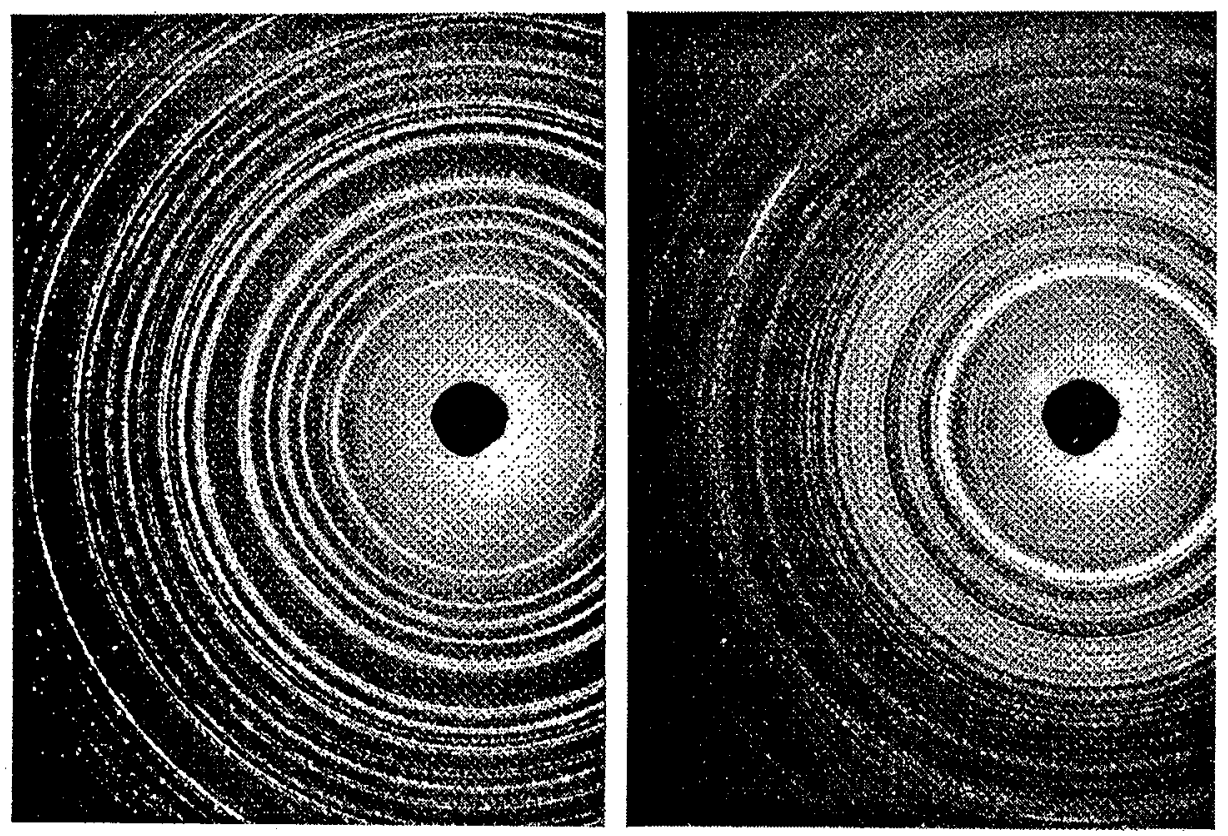

Fig. 1. Typical image plate diffraction patterns of calcite held in the DAC at near ambient conditions (left) and within the metastable stability field of $\mathrm{CaCO}_{3}$-III (right). 


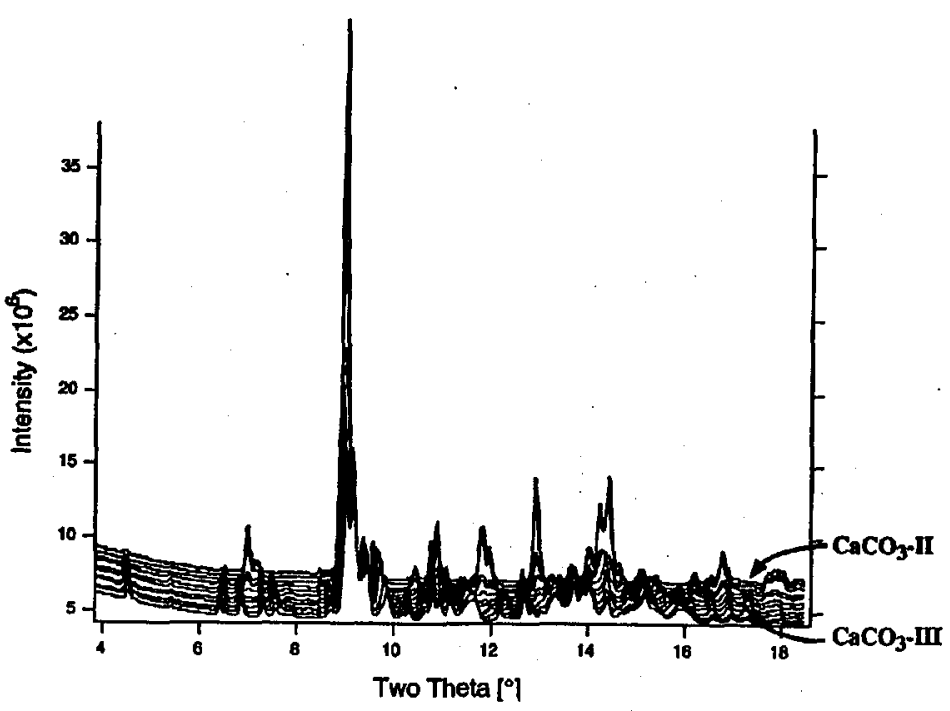

Fig. 2. Pressure dependence of the powder diffraction of $\mathrm{CaCO}_{3}$. Successive diffraction patterns have been offset vertically for clarity, the upper patterns corresponding to monoclinic $\mathrm{CaCO}_{3}$-II (at approximately $1.5 \mathrm{GPa}$ ) which transforms to (apparently lower-symmetry) $\mathrm{CaCO}_{3}$-III on increasing pressure (lower patterns). The complexity of the diffraction patterns of $\mathrm{CaCO}_{3}-\mathrm{III}$ is evident.

to $\mathrm{CaCO}_{3}-\mathrm{II}$ at around $1.45 \mathrm{GPa}$ and further transforms to another metastable polymorph $\left(\mathrm{CaCO}_{3}-\mathrm{III}\right)$ at slightly higher pressures. The space group and structure of $\mathrm{CaCO}_{3}$-III is unknown, although Davis [14] has assigned it an orthorhombic unit cell. The $\mathrm{CaCO}_{3}$ I-II and II-III transitions are both rapid, reversible and unquenchable, and lead to transformation twinning at high- $P$ which has hampered study by single crystal diffraction. The transition of calcite-I to $\mathrm{CaCO}_{3}-\mathrm{II}$ results from two displacements. First, an $11^{\circ}$ rotation in opposite directions of the adjacent $\mathrm{CO}_{3}$ groups along the $c$-axis, and second small antiparallel displacements of adjacent $\mathrm{Ca}$ ions. The $\mathrm{CO}_{3}$ group rotational displacements are thought to drive the transition, just as they drive the high-temperature orientational disordering transition in calcite-I.

Redfern et al. [12] investigated the phase transitions in the metastable calcite polymorphs with the aim of determining the structure of $\mathrm{CaCO}_{3}$-III and the nature of the II-III transition. They used the image plate diffraction system with the hope of determining the high-pressure structures by Rietveld refinement. Thus, it is possible to determine the critical displacement patterns which freeze in at high pressure, and to observe co-elastic strains associated with the transformations at high pressure. Such ferroelastic-related strain measurements and structural studies require the high-resolution and reliable intensity statistics proven already by high-pressure image plate systems such as that at station 9.1 of the Daresbury (UK) SRS. The experiments were conducted at ambient temperature and high pressure, in the pressure range $0.1 \mathrm{MPa}$ to $3 \mathrm{GPa}$. Typical diffraction patterns 
recorded by the image plate are shown in Fig. 1 . So far, it has not proved possible to index the diffraction patterns of $\mathrm{CaCO}_{3}$-III, but the results show that previous assignments of the symmetry of this structure are incorrect, and it appears that the structure is triclinic. Inspection of the powder diffraction patterns shows the complexity of the high-pressure structure and point to why single crystal structural studies of this material have all failed (Fig. 2).

\section{3. "Large volume" high- $P / T$ apparatus}

In contrast to the extreme $P_{\mathrm{s}}$ and $T$ s achievable by laser heated and externally-heated DACs, more modest $P / T$ regimes may be accessed using large volume multi-anvil press devices. The advantage of using such apparatus is that temperature gradients are small, the sample environment $\left(\mathrm{fO}_{2}\right.$ etc.) is potentially easier to control, and a larger polycrystalline sample volume of grains in contact with one another enables inter-mineral reactions to be studied in situ under high- $P / T$ conditions approaching the lithostatic conditions of the Earth's interior. Two-stage multi-anvil apparatuses are capable of attaining pressures and temperatures (fairly routinely) of more than $25 \mathrm{GPa}$ and $2500 \mathrm{~K}$. Conveniently, this corresponds to reaching down to the conditions of the lower mantle. The majority of the in situ studies using multi-anvil presses at synchrotron sources have been focused on elucidating the characteristics of the phase transitions associated with geophysical discontinuities at the top and bottom of the transition zone, ranging from the conditions of the Earth's upper mantle to those of the lower mantle.

In order to obtain diffraction from a sample held in situ in a multi-anvil press it is necessary to have a pathway for $\mathrm{X}$-rays through the apparatus that is not prohibitively absorbing. This is generally achieved by directing the $\mathrm{X}$-rays between the anvils of the apparatus. Three multi-anvil presses have been on-line at synchrotron sources for some time now: MAX-80 and MAX-90 (multi anvil X-ray apparatus designed in 1980 and 1990) at the Photon Factory, Tsukuba, Japan, and SAM85 (six anvil machine designed in 1985) at the NSLS, Brookhaven, USA. These are of a common design, based on an arrangement of six steel anvils which can either be employed to directly squeeze a cubic sample arrangement, as a single-stage device, or alternatively bear upon eight second-stage anvils in a two-stage arrangement. The whole arrangement is pressed within a vertical 250 ton loading frame, the uniaxial force being converted to a quasi-hydrostatic pressure by the arrangement of guide blocks which divides the ram force between the six stage-one steel anvils $[15,16]$. The incoming X-ray beam is horizontal (perpendicular to the ram force) and is diffracted in the vertical plane. Deviatoric stresses can be determined from the diffraction of a standard material pressurised within the cell, and recent studies have shown that it can be characterised quite readily. In high- $P / T$ experiments under constant ram load competing factors which influence the measured pressure include the deviatoric stress (which saturates at some $3 \mathrm{GPa}$ ), the thermal pressure due to the thermal expansion of the sample (which will act to increase $P$ ) and mechanical weakening of the anvils and cell materials on increasing $T$ (which reduces the effective $P$ ) $[17,18]$.

These types of multi-anvil presses have been used for some time now within the Earth Science community for synthesis work and in quench experiments. It is 
unsurprising, therefore, that the majority of the work done at the NSLS has been Earth Science related, much of it associated with the Center in High-Pressure Research. On the other hand, mineralogical studies formed only a minority of the early work done at the Photon Factory. Many [17, 19] of them have been single-stage experiments on the phase relations of the olivine-wadsleyite-ringwoodite phases of the $(\mathrm{Mg}, \mathrm{Fe})_{2} \mathrm{SiO}_{4}$ system; perovskite phases such as $\mathrm{CaSiO}_{3}[18]$, neighborite [20], and $(\mathrm{Mg}, \mathrm{Fe}) \mathrm{SiO}_{3}[21-25]$ have also been investigated using both single-stage and two-stage arrangements. In the latter, one or more of the second-stage cubic anvils is constructed from sintered diamond to allow the transmission of the diffracted beam. The arrangement is, by necessity, energy-dispersive, with the disadvantages in terms of resolution which this brings. Magnesiosilicate perovskite is highly metastable at low pressure, and in situ double-stage multi-anvil experiments allow a route to synthesis of the sample and then the study of it without the danger it back-transforming, as it would in a quench and re-heating process. None the less, the poor resolution of the energy-dispersive system means that it is unlikely that subtle ferroelastic phase transitions in magnesiosilicate perovskite would be easily detected, and it remains to be seen whether this orthorhombic perovskite transforms to tetragonal or cubic under mantle geotherm conditions, as has been suggested by some recent computer simulations.

A new development in the use of large volume apparatuses at synchrotron facilities is the recent installation of a MA6-8 Walker cell [26, 27] at Daresbury (UK) SRS. In this system access holes are drilled through pressure distribution plates at either end of the cylindrical cell, as well as two of the guide wedges. The loading frame is positioned horizontally at $35^{\circ} 16^{\prime}$ to the X-ray beam (Fig. 3). In tnis arrangement the guide wedges move together along the axis of the loading frame, but apart perpendicular to the ram force, stretching the compression ring. Upon compression, therefore, the wedges move apart perpendicular to the path of the X-rays, obviating alignment problems. The incident and diffracted beams

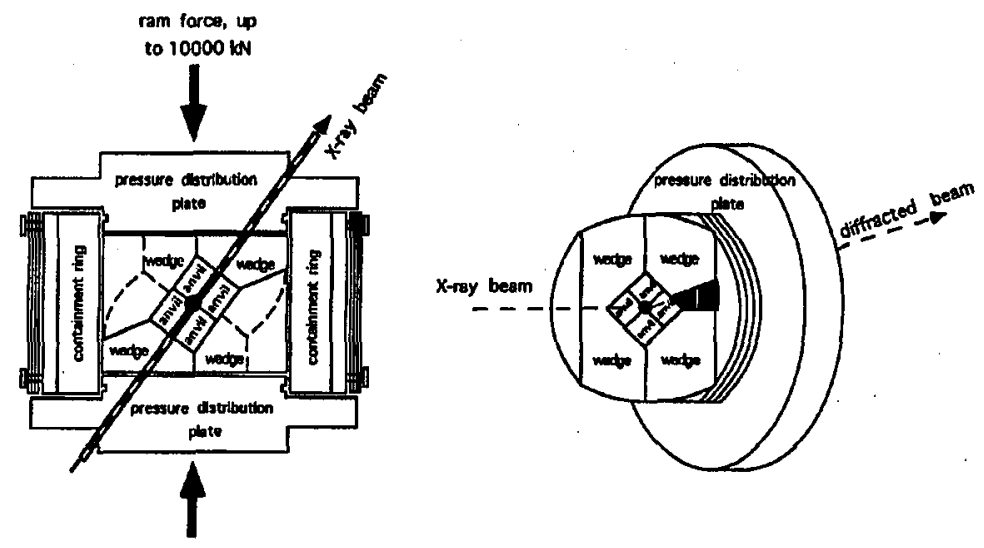

Fig. 3. Top (left) and side (right) view of the MA6-8 Walker cell installed on station 16.4 of Daresbury SRS. 
pass along a central plane between cubic anvils. Initial tests have demonstrated the feasibility of this approach, and the facility has already become available to users.

\section{A final note on deviatoric stress}

Measurements of equilibrium properties of minerals at high $P$ and $T$ require that the experiments be conducted under conditions of hydrostatic (or lithostatic) pressure. In some cases apparent stiffening of materials at high pressure has been observed, and this has led to an active discussion within the mineral physics community regarding the role of non-hydrostaticity [28] and the possibility of overestimate of pressure using the ruby fluorescence technique [29]. Even nominally hydrostatic media such as noble gases may cause problems at very high- $P$ because of their potential solubility into the solid phases of interest, a phenomenon which apparently displays itself as loss of crystallinity on decompression [30].

An alternative approach to the problem of non-hydrostaticity is to make use of the fact that it readily arises, by investigating the properties of mantle materials under imposed shear stresses. Thus, Wu et al. [31] were able to enhance the shear stresses in a DAC experiment by omitting a gasket. They observed the spatial-dependence of transformation of olivine to wadsleyite and ringwoodite as rings of transformation, related to the radially varying degrees of shear stress across their high- $P / T$ diamond cell. They demonstrated that shear stresses enhance the kinetics of transformation of olivine to wadsleyite at low temperatures, a result which has important implications for the mechanisms of deep focus earthquakes in cold subducted oceanic crust. Weidner et al. [32] have similarly made use of deviatoric stress in the SAM85 apparatus, demonstrating that the deviatoric stress in a sample can be measured as a function of pressure, and from its disappearance the yield strength (and its $P$ dependence) may be inferred.

With such ingenuity demonstrated within the high- $P / T$ Earth Science community, it is to be expected that further advances in our understanding of Earth materials under the conditions of the planet's interior will continue to be made, in line with advances in the sophistication of high- $P / T$ apparatus and the properties of synchrotron sources. There are a range of experiments we have not touched upon, including spectroscopic techniques which would be relevant to studies of the melting transition. In addition, much attention has also been focused in recent years on the role of $P$ in amorphising minerals [33]. These experiments will undoubtedly throw up challenges for our models of mineral behaviour at high $P$ and $T$. Perhaps the most significant technological advance which could now be made (one that would considerably enhance our experimental capabilities) would be an improvement in energy-sensitivity of energy-dispersive detectors, or an alternative higher-resolution diffraction geometry which could be employed in large-volume and DAC experiments.

\section{References}

[1] S.A.T. Redfern, B.J. Wood, C.M.B. Henderson, Geophys. Res. Lett. 20, 2099 (1993).

[2] A.R. Pawley, S.A.T. Redfern, B.J. Wood, Mineral. Petrol. 122, 301 (1995). 
[3] T.J.B. Holland, S.A.T. Redfern, A.R. Pawley, Am. Mineral. 81, 341 (1996).

[4] T. Yagi, T. Hishinuma, Geophys. Res. Lett. 22, 1933 (1995).

[5] Y.W. Fei, H.K. Mao, Science 266, 1678 (1994).

[6] H. Harder, U.R. Christensen, Nature 380, 507 (1996).

[7] A.P. Jephcoat, L.W. Finger, D.E. Cox, High Press. Res. 8, 667 (1992).

[8] R.J. Nelmes, M.I. McMahon, P.D. Hatton, R.O. Piltz, J. Crain, R.J. Cernick, G. Bushnell-Wye, High Press. Res. 8, 677 (1992).

[9] W.L. Huang, W.A. Bassett, T.C. Wu, Am. Mineral. 79, 683 (1994).

[10] T.C. Wu, A.H. Shen, M.S. Weathers, W.A. Bassett, Am. Mineral. 80, 941 (1995).

[11] S.K. Saxena, L.S. Dubrovinsky, P. Häggkvist, Y. Cerenius, G. Shen, H.K. Mao, Science 269, 1703 (1996).

[12] S.A.T. Redfern, M.T. Dove, R.J. Nelmes, Daresbury Annual Report, CCLRC, UK, 1996 , in press.

[13] P.W. Bridgman, Am. J. Sci. 37, 7 (1939).

[14] B.L. Davis, Science 145, 489 (1964).

[15] M.T. Vaughan, in: Experiments at High Pressure and Applications to the Earth's Mantle, Ed. R.W. Luth, MAC Short Course Handbook, Vol. 21, Mineralogical Association of Canada, Edmonton (Canada) 1993, p. 95.

[16] D.J. Weidner, M.T. Vauhan, J. Ko, Y. Wang, K. Leinenweber, X. Liu, A. Yeganeh-Haeri, R.E. Pacalo, Y. Zhao, High Press. Res. 8, 617 (1992).

[17] Y. Meng, D.J. Weidner, G.D. Gwanmesia, R.C. Liebermann, M.T. Vaughan, Y. Wang, K. Leinenweber, A. Pacalo, A. Yeganeh-Haeri, Y. Zhao, J. Geophys. Res. 98, 22199 (1993).

[18] Y. Wang, D.J. Weidner, F. Guyor, J. Geophys. Res. 101, 661 (1996).

[19] H. Morishima, T. Kato, M. Suot, E. Ohtani, S. Urakawa, W. Utsumi, O. Shimomura, T. Kikegawa, Science 265, 1202 (1994).

[20] Y.S. Zhao, D.J. Weidner, J.D. Ko, K. Leinenweber, X. Liu, B.S. Li, Y. Meng, R.E.G. Pacalo, M.T. Vaughan, Y.B. Wang, A. Yeganeh-Haeri, J. Geophys. Res. 99, 2871 (1994).

[21] H. Morishima, E. Ohtani, T. Kato, O. Shimomura, T. Kikegawa, Geophys. Res. Lett. 21, 899 (1994).

[22] T. Kondo, H. Sawamoto, A. Yoneda, M. Kato, A. Matsumuro, T. Yagi, T. Kikegawa, Pure Appl. Geophys. 141, 601 (1993).

[23] W. Utsumi, N. Funamori, T. Yagi, E. Ito, T. Kikegawa, O. Shimomura, Geophys. Res. Lett. 22, 1005 (1995).

[24] T. Kato, E. Ohtani, H. Morishima, D. Yamazaki, A. Suzuki, M. Suto, T. Kubo, J. Geophys. Res. 100, 20475 (1995).

[25] N. Funamori, T. Yagi, W. Utsumi, T. Kondo, T. Uchida, M. Funamori, J. Geophys. Res. 101, 8257 (1996).

[26] D. Walker, M.A. Carpenter, C.M. Hitch, Am. Mineral. 75, 1020 (1990).

[27] S.M. Clark, Nucl. Instrum. Methods Phys. Res. A 381, 161 (1996).

[28] T.C. Wu, W.A. Bassett, Pure Appl. Geophys. 141, 509 (1994).

[29] B. Reynard, G. Fiquet, J.P. Itié, D.R. Rubie, Am. Mineral. 81, 45 (1996).

[30] R.T. Downs, C.S. Zha, T.S. Duffy, L.W. Finger, Am. Mineral. 81, 51 (1996). 
[31] T.C. Wu, W.A. Bassett, P.C. Burnley, M.S. Weathers, J. Geophys. Res. 98, 19767 (1993).

[32] D.J. Weidner, Y.B. Wang, M.T. Vaughan, Geophys. Res. Lett. 21, 753 (1994).

[33] S.A.T. Redforn, Mineral. Mag. 60, 493 (1996). 